\title{
PREVALENCIA DE LA PRÁCTICA DE ACTIVIDAD FÍSICA Y ESTADO NUTRICIONAL EN ADOLESCENTES DE LA SUBREGIÓN SANTANDER Y NORTE DE SANTANDER COLOMBIA: RESULTADOS ENSIN 2015.
}

\author{
PREVALENCE OF THE PRACTICE OF PHYSICAL ACTIVITY AND NUTRITIONAL STATUS IN ADOLESCENTS \\ OF THE SANTANDER SUBREGION AND NORTHERN SANTANDER COLOMBIA: ENSIN 2015 RESULTS.
}

Poveda Acelas Carlos Augusto ${ }^{1}$, Poveda Acelas Dana Carolina ${ }^{2}$.

1 Universidad CES, Medellín Colombia. 2 Universidad Jaume I Castellón de la Plana España.

Citation: Poveda Acelas C.A., Poveda Acelas D.C. (2021) Prevalencia de la práctica de actividad física y estado nutricional en adolescentes de la Subregión Santander y Norte de Santander Colombia: resultados ENSIN 2015. Revista Salud Pública y Nutrición, 20 (2), 13-21.

Editor: Esteban G. Ramos Peña, Dr. CS., Universidad Autónoma de Nuevo León, Facultad de Salud Pública y Nutrición, Monterrey Nuevo León, México. Copyright: @2021 Poveda Acelas C.A., et al. This is an open-access article distributed under the terms of Creative Commons Attribution License [CC BY 4.0], which permits unrestricted use, distribution, and reproduction in any medium, provided the original author and source are credited.

Competing interests: The authors have declared that no competing interests exist.

DOI: https://doi.org/10.29105/respyn20.2-2

Recibido: 04 de septiembre 2020; Aceptado: 20 de enero 2021

Email: carlos226@ @hotmail.com 


\title{
PREVALENCIA DE LA PRÁCTICA DE ACTIVIDAD FÍSICA Y ESTADO NUTRICIONAL EN ADOLESCENTES DE LA SUBREGIÓN SANTANDER Y NORTE DE SANTANDER COLOMBIA: RESULTADOS ENSIN 2015.
}

\author{
Poveda Acelas Carlos Augusto ${ }^{1}$, Poveda Acelas Dana Carolina².
}

1 Universidad CES, Medellín Colombia. 2 Universidad Jaume I Castellón de la Plana España.

\section{RESUMEN}

Introducción. La inactividad física en adolescentes es un problema de salud pública a nivel mundial, pues contribuye al desarrollo de sobrepeso, obesidad y enfermedades crónicas. Objetivo: Describir la prevalencia de la práctica de actividad física y el estado nutricional de los adolescentes (13 a 17 años) residentes en la Subregión: Santander y Norte de Santander de Colombia que participaron en la última Encuesta Nacional de la Situación Nutricional de Colombia (ENSIN 2015). Métodos: Estudio descriptivo de corte transversal, de fuente secundaria, proveniente de 847 registros de la Encuesta Nacional de la Situación Nutricional. El análisis descriptivo consistió en el cálculo de frecuencias absolutas y relativas debido a que las variables del estudio se consideraron de naturaleza cualitativa. Resultados: El 14.8\% de los adolescentes cumplían los 60 minutos diarios de actividad física recomendados por la Organización Mundial de la Salud (OMS). En cuanto a la actividad física según el estado nutricional se encontró que los adolescentes menos activos físicamente fueron aquellos con sobrepeso (13.0\%) y obesidad (2.9\%). Conclusiones: Se requiere fomentar la actividad física diaria en los adolescentes para evitar las consecuencias físicas, psicológicas y sociales que genera no realizarla.

Palabras Clave: Adolescente, actividad física, estado nutricional.

\section{ABSTRACT}

Introduction: Physical inactivity in adolescents is a public health problem worldwide, as it contributes to the development of overweight, obesity and chronic diseases. Objective: Describe the prevalence of physical activity practice, and nutritional status in adolescents ( 13 to 17 years old) from the Subregion: Santander and Norte de Santander of Colombia who participated in the last National Survey of the Nutritional Situation of Colombia (ENSIN 2015). Material and methods: Descriptive crosssectional study, secondary source, from 847 records of the National Survey of the Nutritional Situation. The descriptive analysis consisted of the calculation of absolute and relative frequencies because the study variables were considered qualitative in nature. Results: $14.8 \%$ of adolescents fulfilled the 60 minutes of physical activity per day recommended by the World Health Organization (WHO). Regarding physical activity according to nutritional status, it was found that the least physically active adolescents were those with overweight (13.0\%) and obesity (2.9\%). Conclusions: It is necessary to promote daily physical activity in adolescents, given the physical, psychological and social consequences of not doing it.

Key words: adolescent, exercise, nutritional status. 


\section{Introducción}

La actividad física es definida por la Organización Mundial de la Salud (OMS) como toda acción que involucra un trabajo muscular y aumento del gasto energético (OMS, 2020). Diferentes estudios han mostrado que la realización constante de actividad física durante la infancia y adolescencia tiene un impacto positivo en el crecimiento y desarrollo físico, social y psicológico de los menores de edad; además que la práctica regular de actividad física desde estas etapas de la vida fomenta estilos de vida saludables en la edad adulta, convirtiéndose en factores claves para un buen estado nutricional y de salud (Slowik et al., 2019).

Expertos en el mundo han señalado cómo la práctica regular de actividad física tiene múltiples beneficios para la salud de los adolescentes; dentro de ellos destacan: incrementa la capacidad aeróbica, la composición corporal, la aptitud muscular, estimula el crecimiento de la autoestima, la socialización, mejora el rendimiento intelectual; además previene diversas enfermedades crónicas como son: diabetes, obesidad, enfermedades cardiovasculares (Mikaelsson, Rutberg, Lindqvist y Michaelson, 2019; Sánchez et al.,2019).

No obstante, pese a estos beneficios, realizar actividad física no es un hábito común en la población adolescente (Del Ciampo, Louro, Del Ciampo y Ferraz, 2019); lo cual ha desencadenado que las cifras de sobrepeso y obesidad en este grupo poblacional estén aumentando cada vez más (Chen et al., 2020).

A nivel mundial se ha encontrado que la inactividad física es un problema de salud pública, dado que contribuye al desarrollo de obesidad, enfermedades cardiovasculares y metabólicas (Whooten, Kerem y Stanley, 2019). Según la Organización Mundial de la Salud (OMS) los adolescentes deben realizar al menos 60 minutos diarios de actividad física moderada a intensa para que se le consideren como físicamente activos y gocen de los beneficios para la salud que dicha práctica genera (Organización Mundial de la Salud [OMS], 2020). Sin embargo, actualmente se estima que en el mundo el $80 \%$ de los adolescentes son inactivos físicamente (García, 2019).
En Latinoamérica y el Caribe, un estudio realizado con datos que contenían información sobre la actividad física de los adolescentes de 26 países durante 2007-2013, reportó que solo el $15 \%$ de los adolescentes cumplían con las recomendaciones de actividad física; en países como San Vicente y Granadinas ( $12.8 \%$ de los menores de edad son activos físicamente), en Antigua y Barbados (22.8\%), Argentina (16.7\%), Bolivia (13.9\%), Chile (13.6\%), Costa Rica (18.5\%), Perú (15.4\%), Ecuador $(14.5 \%)$ y Guatemala (15.6\%); Además existe evidencia que en cuanto a la actividad física por sexo; los hombres son más activos físicamente que las mujeres (Farias et al., 2018).

En México la Encuesta Nacional de Salud y Nutrición 2018-2019 (ENSANUT) reportó que el $53.7 \%$ de los adolescentes cumplen con la recomendación de actividad física diaria. Los resultados por sexo de la ENSANUT 2018-2019 muestran que el $46.5 \%$ de las mujeres y $60.9 \%$ de los hombres, cumple con la indicación dada por la OMS (Shamah et al., 2020).

Naciones como Antigua y Barbados describieron que solo el $28.5 \%$ de los hombres y $16.7 \%$ de las mujeres cumplían con las recomendaciones de actividad física propuestas por la OMS; en Argentina el 21.7 $\%$ de los hombres y $12.1 \%$ de las mujeres; en Chile $18.3 \%$ de los hombres y $9.3 \%$ de las mujeres y en Ecuador $18.0 \%$ de los hombres y $10.8 \%$ de las mujeres (Farias et al., 2018).

En el contexto colombiano, la Encuesta Nacional de la Situación Nutricional (ENSIN) encuesta que es realizada en el país cada 5 años y cuya última versión fue en el año 2015, permitió referenciar la situación nutricional de la población colombiana, describiendo para el 2015 que el $13.4 \%$ de los adolescentes de Colombia cumplían con las recomendaciones de actividad física (Encuesta Nacional de la Situación Nutricional en Colombia [ENSIN], 2015a).

No obstante, en la Subregión de Santander y Norte de Santander de Colombia no existen estudios que reporten la prevalencia de las prácticas de actividad física y estado nutricional en adolescentes.

Debido a lo anterior, este estudio tuvo como objetivo: describir la prevalencia de la práctica de actividad física y el estado nutricional de los adolescentes (13 
a 17 años) residentes en la Subregión: Santander y Norte de Santander de Colombia, que participaron en la Encuesta Nacional de la Situación Nutricional de Colombia 2015.

\section{Materiales y Métodos}

Se presenta un estudio cuantitativo, descriptivo de corte transversal, de fuente de información secundaria, en el que se hizo uso de la estadística univariada (descriptiva) para el análisis de la información.

La información de este estudio se obtuvo de la ENSIN 2015, la cual fue suministrada por el Ministerio de Salud y Protección Social de Colombia. La ENSIN 2015 es una encuesta nacional en torno a la situación nutricional de la población colombiana. El desarrollo de la encuesta fue liderado por el Instituto Colombiano de Bienestar Familiar en coordinación del Ministerio de Salud y Protección Social, el Instituto Nacional de Salud y el Departamento Administrativo para la Prosperidad Social y tuvo como objetivo analizar la situación alimentaria y nutricional de la población colombiana de acuerdo a los determinantes sociales definidos por la ENSIN (Encuesta Nacional de la Situación Nutricional en Colombia [ENSIN], 2015, p.2).

\section{Población estudiada y muestra}

Teniendo en cuenta que la ENSIN 2015 se encuentra segmentada en grupos de edad, para efectos de esta investigación la unidad de observación fueron los registros que se encontraban en la base de datos de la ENSIN 2015 correspondientes a adolescentes entre los 1317 años que residían en la Subregión: Santander y Norte de Santander.

De acuerdo con lo anterior, se tuvo una población de 889 datos de adolescentes de la subregión, los cuales fueron depurados eliminando aquellos que estaban incompletos o que tenían variables con datos inconsistentes (aquellas donde no concordaba la información), quedando finalmente 847 registros para el análisis.

De otro lado, en esta investigación se estudiaron 3 componentes que hicieron parte de la ENSIN 2015: condiciones socioeconómicas, antropométrico y de actividad física.
En el primer componente la ENSIN indagó aspectos como el área de residencia de los adolescentes, nivel de escolaridad, edad, sexo, etnia, afiliación al sistema de seguridad social, entre otros.

En el de antropometría la ENSIN realizó en cada menor de edad la medición de talla y peso por personal previamente capacitado y entrenado, posterior a la toma de las medidas antropométricas se pudo establecer el Índice de Masa Corporal para la edad (IMC/ Edad).

Finalmente, para el componente de actividad física la ENSIN 2015 aplicó el cuestionario del Louth Behavior Surveillance System (YRBSS) con el fin de estimar las recomendaciones de actividad física de la población adolescente (Encuesta Nacional de la Situación Nutricional en Colombia[ENSIN], 2015, p. 41) Este es un cuestionario internacional de aproximadamente 86 preguntas, el cual indaga sobre comportamientos alimentarios poco saludables de los adolescentes y actividad física inadecuada (Centers for Disease Control and Prevention [CDC], 2019).

Dentro de las preguntas de actividad física que se midieron en la ENSIN y que se tuvieron en cuenta en esta investigación se encuentran:1. De los siete días a la semana ¿cuántos días realizó actividad física?, 2. De los siete días a la semana, ¿cuántos días llevó a cabo ejercicios de levantamiento de pesas?, 3. ¿En dónde usted vive hay presencia de parques o centros deportivos para realizar actividad física? pregunta que se realizó para determinar la disponibilidad y acceso a lugares para realizar física en los lugares de residencia, 4. ¿Va a ciclovías los fines de semana para realizar actividad física? y 5 . ¿Asiste a escuelas deportivas?

\section{Variables del estudio:}

Este estudio tiene 10 variables, las cuales están distribuidas en tres grupos: sociodemográficas, estado nutricional y de actividad física. Las variables sociodemográficas del estudio fueron: lugar de residencia, edad del adolescente, sexo y nivel de escolaridad. La variable estado nutricional fue medida a través del Índice de Masa Corporal para la edad. El grupo de variables de actividad física estuvo conformado por cinco: 1 . Activo físicamente durante 60 minutos diarios, 2. Ejercicios de levantamiento de pesas en la semana, 3. Presencia de parques o centros 
deportivos en el lugar de residencia para realizar actividad física. 4. Acude a ciclovías los fines de semana a realizar actividad física, 5. Asistencia a escuelas deportivas para realizar actividad física en la semana.

La práctica de actividad física fue determinada a partir de las variables: Ejercicios de levantamiento de pesas en la semana, presencia de parques o centros deportivos en el lugar de residencia para realizar actividad física, acude a ciclovías los fines de semana a realizar actividad física y asistencia a escuelas deportivas para realizar actividad física en la semana.

Para determinar la prevalencia de actividad física se tuvo en cuenta los datos de la variable activo físicamente durante 60 minutos diarios, ya que el parámetro de la OMS para identificar a un adolescente físicamente activo es: realizar actividad física moderada a intensa por 60 minutos los 7 días de la semana. De esta manera, solo los adolescentes que realizaban 60 minutos de actividad física diaria fueron considerados como físicamente activos.

El estado nutricional de los adolescentes se estableció de acuerdo a la directriz dada por la Resolución 2465 de 2016 del Ministerio de Salud y Protección Social de Colombia, donde se enuncia que el índice de Masa Corporal/edad es el indicador idóneo para establecer el estado nutricional de los adolescentes. Se tomaron las desviaciones estándar (DE) del IMC que estaban en la base de datos y se procedió a clasificar el estado nutricional de los adolescentes en las siguientes categorías: sobrepeso a aquellos menores de edad cuyo IMC /edad ( $>+1$ a $+\leq 2 \mathrm{DE})$, obesidad aquellos adolescentes en $(>+2$ DE), IMC adecuado para la edad DE entre $(\geq-1 \mathrm{a} \leq$ $+1 \mathrm{DE})$, riesgo de delgadez $(\geq-2 \mathrm{a} \leq-1 \mathrm{DE}) \mathrm{y}$ delgadez $(<-2 \mathrm{DE})$.

Para el procesamiento y análisis de la información se usó el programa SPSS versión 21 licencia Universidad CES. El análisis descriptivo requirió el cálculo de frecuencias absolutas y relativas, ya que para fines de esta investigación todas las variables de este estudio fueron categorizadas como de naturaleza cualitativa.

\section{Consideraciones éticas}

La investigación contó con la autorización del Ministerio de Salud y Protección de salud de
Colombia para el uso de la base de datos, también tuvo en cuenta lo expresado por la declaración de Helsinki y las pautas de investigación en humanos, además obtuvo el aval de comité de ética de la Universidad CES Medellín Colombia (acta 151 del comité de ética en investigación en humanos Universidad CES).

\section{Resultados}

La tabla 1 presenta las características sociodemográficas de los 847 registros de adolescentes estudiados según sexo, en donde se puede observar que más del $70 \%$ de los y las adolescentes vivían en la cabecera municipal. En lo que respecta al nivel de escolaridad se puede aseverar que más del $85 \%$ de los participantes del estudio tenían entre primaria completa y secundaria incompleta.

\begin{tabular}{|c|c|c|c|c|c|}
\hline & & \multicolumn{4}{|c|}{ Sexo del adolescente } \\
\hline \multirow{2}{*}{\multicolumn{2}{|c|}{ Caracteristicas sociodemográficas }} & \multicolumn{2}{|c|}{ Masculino } & \multicolumn{2}{|c|}{ Femenino } \\
\hline & & No. & $\%$ & No. & $\%$ \\
\hline \multirow{3}{*}{ 1. Lugar de residencia } & Cabecera municipal & 330 & 72.8 & 304 & 77.2 \\
\hline & Centro poblado & 12 & 2.6 & 10 & 2.5 \\
\hline & Rural disperso & 111 & 24.5 & 80 & 20.3 \\
\hline \multirow{3}{*}{ 2. Edad } & Entre 13 a 15 años & 272 & 60.0 & 220 & 55.8 \\
\hline & Entre 16 a 17 años & 181 & 40.0 & 174 & 44.2 \\
\hline & Menos de primaria completa & 20 & 4.4 & 11 & 2.8 \\
\hline \multirow{2}{*}{$\begin{array}{l}\text { 3. Nivel de escolaridad } \\
\text { del adolescente }\end{array}$} & $\begin{array}{l}\text { Entre primaria completa y } \\
\text { secundaria incompleta }\end{array}$ & 391 & 86.3 & 339 & 86.0 \\
\hline & $\begin{array}{l}\text { Entre secundaria completa y } \\
\text { superior incompleta }\end{array}$ & 42 & 9.3 & 44 & 11.2 \\
\hline
\end{tabular}

La tabla 2 indica la prevalencia de actividad física de los adolescentes, donde se puede aseverar que el 14.8 $\%$ de los menores de edad eran activos físicamente, lo cual indica que el $85.2 \%$ de los menores de edad eran inactivos físicamente, es decir no realizaban mínimo 60 minutos de actividad física los 7 de días de la semana. 
Tabla 2. Prevalencia de actividad física de los adolescentes de la subregión: Santander y Norte de Santander. ENSIN, 2015

\begin{tabular}{cccc}
\hline \multicolumn{2}{c}{ Actividad física } & No. & $\%$ \\
\hline & 0 días & 216 & 25.5 \\
Activo & 1 día & 177 & 20.9 \\
físicamente & 2 días & 123 & 14.5 \\
durante 60 & 3 días & 89 & 10.5 \\
minutos & 4 días & 51 & 6.0 \\
diarios & 5 días & 44 & 5.2 \\
& 6 días & 22 & 2.6 \\
& 7 días & 125 & 14.8 \\
\hline
\end{tabular}

Fuente: Elaboración propia en base a datos de la ENSIN 2015 proporcionados por el Ministerio de Salud y Protección Social de Colombia.

La tabla 3 señala la actividad física de los sujetos del estudio según sexo, en ella se observa que los hombres fueron más activos físicamente $(20.5 \%)$ que las mujeres (8.1\%). También muestra que los hombres realizan en mayor porcentaje ejercicios que involucran el levantamiento de pesas. Para la variable asistencia a escuelas deportivas, la tabla presenta que el $63.6 \%$ de los hombres van a este tipo de centros deportivos, mientras que las mujeres van en un $57.1 \%$.

Además, la tabla indica que más del $70 \%$ de los y las adolescentes tenía en los lugares de residencia parques o centros deportivos.

\begin{tabular}{|c|c|c|c|c|c|}
\hline \multirow{3}{*}{ Actividad física } & & \multicolumn{4}{|c|}{ Sexo del adolescente } \\
\hline & & \multicolumn{2}{|c|}{ Masculino } & \multicolumn{2}{|c|}{ Femenino } \\
\hline & & No. & $\%$ & No. & $\%$ \\
\hline \multirow{8}{*}{$\begin{array}{l}\text { 1. Activo físicamente durante } 60 \\
\text { minutos diarios }\end{array}$} & 0 días & 78 & 17.2 & 138 & 35.0 \\
\hline & 1 día & 81 & 17.9 & 96 & 24.4 \\
\hline & 2 días & 73 & 16.1 & 50 & 12.7 \\
\hline & 3 días & 47 & 10.4 & 42 & 10.7 \\
\hline & 4 días & 36 & 7.9 & 15 & 3.8 \\
\hline & 5 días & 33 & 7.3 & 11 & 2.8 \\
\hline & 6 días & 12 & 2.6 & 10 & 2.5 \\
\hline & 7 días & 93 & 20.5 & 32 & 8.1 \\
\hline \multirow{8}{*}{ 2. Ejercicios con pesas } & 0 días & 203 & 44.8 & 225 & 57.1 \\
\hline & 1 día & 72 & 15.9 & 74 & 18.8 \\
\hline & 2 días & 69 & 15.2 & 41 & 10.4 \\
\hline & 3 días & 30 & 6.6 & 20 & 5.1 \\
\hline & 4 días & 15 & 3.3 & 5 & 1.3 \\
\hline & 5 días & 12 & 2.6 & 6 & 1.5 \\
\hline & 6 días & 6 & 1.3 & 4 & 1.0 \\
\hline & 7 días & 46 & 10.2 & 19 & 4.8 \\
\hline 3. Presencia de parques o centros & $\mathrm{Si}$ & 349 & 77 & 281 & 71.3 \\
\hline deportivos en lugar de residencia & No & 104 & 23 & 113 & 28.7 \\
\hline \multirow{2}{*}{$\begin{array}{l}\text { 4. Va a ciclovías los fines de semana a } \\
\text { realizar actividad física }\end{array}$} & $\mathrm{Si}$ & 232 & 51.2 & 208 & 52.8 \\
\hline & No & 221 & 48.8 & 186 & 47.2 \\
\hline \multirow{2}{*}{ 5. Asistencia a escuelas deportivas } & $\mathrm{Si}$ & 288 & 63.6 & 225 & 57.1 \\
\hline & No & 165 & 36.4 & 169 & 42.9 \\
\hline
\end{tabular}

Fuente: Elaboración propia en base a datos de la ENSIN 2015 proporcionados por el Ministerio de Salud y Protección Social de Colombia.

En la tabla 4 se presenta el estado nutricional de los sujetos del estudio de acuerdo con el sexo En ella se puede apreciar que más del $60 \%$ de los y las adolescentes presentaban un IMC/Edad adecuado, en lo concerniente a la malnutrición por exceso se puede ver que la obesidad fue más prevalente en los hombres que en las mujeres $(4.9 \%$ y $3.3 \%$ respectivamente); mientras que el sobrepeso fue mayor en las mujeres que en los hombres $(18.5 \%$ y $14.3 \%$ respectivamente). En relación a la malnutrición por déficit, la tabla describe que el porcentaje de riesgo de delgadez y delgadez fue mayor en el sexo masculino.

Tabla 4. Estado nutricional de los adolescentes de la subregión: Santander y Norte de Santander según sexo. ENSIN, 2015.

\begin{tabular}{lcccc}
\hline & \multicolumn{4}{c}{ Sexo del adolescente } \\
& Masculino & \multicolumn{2}{c}{ Femenino } \\
Estado nutricional & No. & $\%$ & No. & $\%$ \\
\hline Obesidad & 22 & 4.9 & 13 & 3.3 \\
Sobrepeso & 65 & 14.3 & 73 & 18.5 \\
IMC Adecuado para la edad & 276 & 60.9 & 273 & 69.3 \\
Riesgo de delgadez & 77 & 17 & 31 & 7.9 \\
Delgadez & 13 & 2.9 & 4 & 1.0 \\
\hline Fuente: Elaboración propia en base a datos de la ENSIN 2015 proporcionados
\end{tabular}

por el Ministerio de Salud y Protección Social de Colombia. 
La tabla 5 describe la actividad física de los adolescentes según el estado nutricional. Se puede ver que los adolescentes que eran físicamente activos tenían mayor porcentaje de riesgo de delgadez (21.3\%) y delgadez (17.6\%), seguido de aquellos con un IMC adecuado (14.6\%). Para los adolescentes con sobrepeso la prevalencia de menores de edad físicamente activos fue de $13.0 \%$ y para los que se encontraban en obesidad de $2.9 \%$.

Para la variable: ejercicios con pesas, se muestra que los adolescentes con sobrepeso e IMC adecuado para la edad fueron los que en mayor porcentaje realizaban ejercicios de fuerza durante los días de la semana. En lo concerniente a la asistencia a escuelas deportivas, la tabla describe que los adolescentes con obesidad son los que en menor porcentaje asistían a centros deportivos (45.7\%).

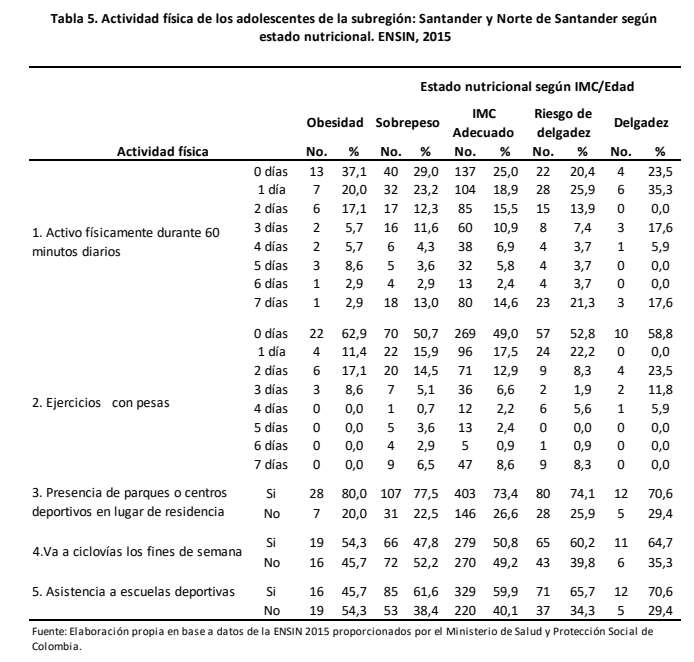

\section{Discusión}

Esta investigación permitió describir la prevalencia de la práctica de actividad física y estado nutricional de los adolescentes de la subregión de Santander y Norte de Santander Colombia. Los resultados mostraron que solo el $14.8 \%$ de los menores de este estudio cumplían con las recomendaciones diarias de actividad física establecida por la OMS (60 minutos de actividad física todos los días para ser considerado activo físicamente), lo cual indicó que el $85.2 \%$ de la población era inactiva físicamente; coincidiendo con lo reportado por un estudio mexicano, donde más del $80 \%$ de los adolescentes no realizaban actividad física (Medina, Jáuregui, Campos y Barquera, 2018). Así mismo, otros estudios realizados en Latinoamérica y a nivel mundial concluyeron que más del $70 \%$ de adolescentes eran inactivos físicamente (Rodríguez y Santos, 2017; Sharma, Cosme y Woo, 2018; Thani et al., 2018; Guthold, Stevens, Riley y Bull, 2020).

Referente a la actividad física según sexo, este estudio encontró que los hombres eran más activos físicamente que las mujeres, encontrando resultados afines a los descritos por investigaciones realizados a nivel mundial, en los que las mujeres eran quienes realizaban menor actividad física en comparación que los hombres (Mera et al., 2017; Guthold et al., 2020; Darling et al., 2020; Colley et al., 2017; Rosselli et al., 2020).

En lo que respecta a las prácticas de actividad física, se halló que los hombres realizan más actividades de fuerza como levantamiento de pesas, además asisten con más frecuencia a escuelas deportivas en comparación que las mujeres, encontrando resultados paralelos con lo descrito por un estudio español en el cual los hombres eran quienes más actividades de musculación $(5,9 \%$ los hombres y $0,5 \%$ las mujeres) y actividades deportivas realizaban $(89 \%$ los hombres frente a $62,8 \%$ las mujeres) (Oliveira y Parra, 2018).

En lo concerniente al estado nutricional de los adolescentes según sexo, los resultados mostraron que más del $60 \%$ de los menores de edad tenían un IMC adecuado para la edad, de igual forma, la obesidad fue más prevalente en los hombres frente a las mujeres, encontrando similitudes con lo expuesto por un estudio realizado en jóvenes, donde más del $50 \%$ de los adolescentes tenían un estado nutricional adecuado, además que los hombres eran quienes mayor obesidad tenían $(3.25 \%$ hombres con obesidad; $1.60 \%$ mujeres con obesidad) (Glinkowska y Glinkowski, 2018).

En relación a la actividad física y el estado nutricional, los resultados de este estudio arrojaron que los adolescentes con riesgo de delgadez $(21.3 \%)$ y delgadez (17.6\%) eran los más físicamente activos, mientras que los menos activos fueron los menores con sobrepeso (13.0\%) y obesidad (2.9\%); encontrando semejanzas con lo reportado por un estudio de Paraguay, donde hallaron que las personas 
con obesidad (26.3\%) y sobrepeso (46.3\%) eran los menos activos físicamente (Ruiz y Sánchez, 2017).

Los anteriores datos reflejan la necesidad de crear diferentes estrategias e intervenciones encaminadas a fortalecer la práctica diaria de actividad física en los adolescentes, haciendo énfasis en los menores de edad con exceso de peso y sexo femenino, dado que son quienes menos actividad física realizaban en la subregión, lo cual los hace más vulnerables al desarrollo de enfermedades crónicas (Di Cesare et al., 2019).

Dentro de las fortalezas de este estudio destacan que es la primera investigación realizada en la subregión de Santander y Norte de Santander sobre prevalencia de la práctica de actividad física y estado nutricional, lo cual lo convierte en una base para futuras investigaciones en la subregión en las que se creen estrategias de intervención orientadas a fomentar la actividad física y mejorar el estado nutricional de los y las adolescentes.

Este estudio presenta ciertas limitaciones, una de ellas es que para medir la actividad física de los adolescentes se utilizó el cuestionario Youth Behavior Surveillance System (YRBSS), el cual no había sido validado en Colombia, sin embargo, fue adaptado para poder ser aplicado en la población adolescente de Colombia. Otra limitación del estudio es que los autores de esta investigación no pueden precisar la calidad de la valoración antropométrica realizada a los menores de edad debido a que no estuvieron presentes en dicha evaluación; sin embargo, se aclara que la ENSIN dentro de su proceso metodológico capacitó al personal que hizo las mediciones. También faltaron otras variables que pudieran aportar información sobre las prácticas de actividad física de los adolescentes como tipos de deportes practicados, así como también variables relacionadas con la alimentación de los adolescentes.

Debido a lo anterior, se recomienda realizar otros estudios que indaguen aspectos de la actividad física como: preferencias de actividad física, razones por las cuales no realizan actividad física (a pesar de contar con espacios deportivos para la práctica deportiva), así mismo sería importante investigar sobre hábitos alimentarios en adolescentes y comportamientos sedentarios (tiempo que pasan los adolescentes frente a pantallas de televisores, computadores y videojuegos).

\section{Conclusiones}

En la subregión de Santander y Norte de Santander Colombia, se encontró que el $14,8 \%$ de los adolescentes eran activos físicamente, siendo los hombres los que en mayor proporción cumplían con la recomendación diaria de actividad física de la OMS $(20,5 \%)$ con relación a las mujeres $(8,1 \%)$. Además, los hombres son los que en mayor porcentaje realizaban prácticas de actividad física como levantamiento de pesas y asistencia a escuelas deportivas.

Por otro lado, los registros estudiados de los menores de edad con sobrepeso y obesidad que participaron en este estudio, son los que en menor proporción realizaban actividad física todos los días.

En consecuencia, es necesario que en la subregión de Santander y Norte de Santander se implementen programas de actividad física orientados a la población adolescente femenina y masculina, pues aunque el sexo masculino fue el que en mayor porcentaje se consideró físicamente activo, las cifras de inactividad física para ambos sexos son altas, de manera que se deben realizar planes de ejercicio físico, así como también es importante promover los beneficios a nivel físico, psicológico y social que genera la práctica de actividad física regular. Igualmente, es fundamental la creación de planes de intervención que mejoren el estado nutricional de los adolescentes.

\section{Agradecimientos}

Al Ministerio de Salud y Protección Social por facilitar la fuente de información.

\section{Bibliografía}

Centers for Disease Control and Prevention. (2019). Youth Risk Behavior Surveillance System (YRBSS). Recuperado de: https://www.cdc.gov/healthyyouth/data/yrbs/ind ex.htm

Chen, P., Wang, D., Shen, H., Yu, L., Gao, Q., Mao, L., Jiang, F., Li, F. (2020). Physical activity and health in Chinese children and adolescents: expert consensus statement. British Journal of 
Sports Medicine, 0,1-11. doi: https://bjsm.bmj.com/content/54/22/1321

Colley, R., Carson, V., Garriguet, D., Janssen, I., Roberts, K., y Tremblay, M. (2017). Physical activity of Canadian children and youth, 2007 to 2015. Health Reports, 28(10), 8-16. Recuperado de: https://pubmed.ncbi.nlm.nih.gov/29044441/

Darling, A., Sunguya, B., Ismail, A., Manu, A., Assefa, N., Sie, A., y Guwattude, D. (2020). Gender differences in nutritional status, diet and physical activity among adolescents in eight countries in sub-Saharan Africa. Tropical Medicine and International Health, 25(1), 33-43. doi: https://doi.org/10.1111/tmi.13330

Del Ciampo, L., Louro, A., Del Ciampo, I., y Ferraz, I. (2019). Sedentary lifestyle among adolescents living in the city of Ribeirao Preto (SP). Journal of Human Growth and Development, 29(3), 403409. doi: https://doi.org/10.7322/jhgd.v29.9539

Di Cesare, M., Soric, M., Bovet, P., Miranda, J., Bhutta, Z., Stevens, G., Bentham, J. (2019). The epidemiological burden of obesity in childhood: a worldwide epidemic requiring urgent action. BMC Medicine, 17(212), 1-20. doi: https://doi.org/10.1186/s12916-019-1449-8

Encuesta Nacional de la Situación Nutricional en Colombia (2015a). Recuperado de: https://www.icbf.gov.co/sites/default/files/ensin 2015 final.pdf

Encuesta Nacional de la Situación Nutricional en Colombia. (2015). Encuesta Nacional de la Situación Nutricional en Colombia-ENSIN. Recuperado de: https://www.minsalud.gov.co/sites/rid/Lists/Bibl iotecaDigital/RIDE/VS/ED/GCFI/documentometodologico-ensin-2015.pdf

Farias, N., Fuentealba, P., Carcamo, J., Cortinez, A., Cristi, C., Von, A., y Sadarangani, K. (2018). A regional vision of physical activity, sedentary behaviour and physical education in adolescents from Latin America and the Caribbean: results from 26 countries. International Journal of Epidemiology, 47(3), 976-986. doi: https://doi.org/10.1093/ije/dyy033
García, F. (2019). Sedentarismo en niños y adolescentes: Factor de riesgo en aumento. Revista Científica Mundo de la Investigación y el Conocimiento, 3(1), 1602-1624. doi: http://www.recimundo.com/index.php/es/article/ view/449

Glinkowska, B., y Glinkowski, W. (2018). Association of sports and physical activity with obesity among teenagers in Poland. International Journal of Occupational Medicine and Environmental Health, 31(6), 771-782.doi: https://doi.org/10.13075/ijomeh.1896.01170

Guthold, R., Stevens, G., Riley, L., y Bull F. (2020). Global trends in insufficient physical activity among adolescents: a pooled analysis of 298 population - based surveys with 1.6 million participants. The Lancet Child \& Adolescent Health, 4(1), 23-35. doi: https://doi.org/10.1016/S2352-4642(19)30323-2

Medina, C., Jáuregui, A., Campos, I., Y Bárquera, S. (2018). Prevalencia y tendencias de actividad física en niños y adolescentes: resultados de Ensanut 2012 y Ensanut MC 2016. Salud Pública de México, 60(3), 263-271. doi: https://doi.org/10.21149/8819

Mera, R., Mera, I., Fornos, J., García, P., Fernández, M., Rodríguez, A., Rica, I. (2017). Análisis de hábitos nutricionales y actividad física de adolescentes escolarizados. RIVACANGAS. Revista Española de Nutrición Comunitaria, 23(1), 1-12. Recuperado de:

http://www.renc.es/imagenes/auxiliar/files/RENC_2 017_1_01. Fornos

Perez JA. Habitos nutricionales_y actividad fisica_adolescentes_RIVACANGAS.pdf

Mikaelsson, K., Rutberg, S., Lindqvist, A., y Michaelson P. (2019). Physically inactive adolescents experiences of engaging in physical activity. European Journal of Physiotherapy, 22(4), 191-196. doi: https://doi.org/10.1080/21679169.2019.1567808

Oliveira, V., y Parra, J. (2018). Género y práctica de ejercicio físico de adolescentes y universitarios. 
Artigos, 48(170), 1114-1128. doi: https://doi.org/10.1590/198053145588

Organización Mundial de la Salud. (2020). Estrategia mundial sobre régimen alimentario, actividad física y salud. Recuperado de: https://www.who.int/dietphysicalactivity/pa/es/

Rodrigues, T., y Santos, D. (2017). Prevalence of physical activity among adolescents in southern Brazil. Journal of Bodywork and Movement Therapies, 22(1), 57-63. doi: https://doi.org/10.1016/j.jbmt.2017.03.022

Roselli, M., Ermini, E., Tosi, B., Boddi, M., Stefani, L., Toncelli, L., y Modesti, P. (2020). Gender differences in barriers to physical activity among adolescents. Nutrition, Metabolism and Cardiovascular Diseases, 30(9), 1582-1589. doi: https://doi.org/10.1016/j.numecd.2020.05.005

Ruiz, S., y Sánchez, S. (2017). Actividad física y estado nutricional en adolescentes de 9 a 15 años de una institución de enseñanza de San Lorenzo, Paraguay. Pediatria (Asunción), 44(2), 111-116. doi:

http://dx.doi.org/10.18004/ped.2017.agosto.111116

Sánchez, Francisco., Campos, A., Vega, Marina., Cortés, Olga., Esparza, María., Sánchez, José.,...Mengual, José. (2019). Promoción de la actividad física en la infancia y la adolescencia (parte 1). Pediatría Atención Primaria, 21(83), 279-291. Recuperado de: http://scielo.isciii.es/scielo.php?script=sci_arttex $\underline{\text { t\&pid}=S 1139-76322019000300019}$

Shamah, T., Vielma, E., Heredia, O., Romero, M., Mojica, J., Cuevas, L., Santaella, J., y Rivera, J. (2020). Encuesta Nacional de Salud y Nutrición 2018-19: Resultados Nacionales. Cuernavaca, México: Instituto Nacional de Salud Pública. Recuperado de: https://ensanut.insp.mx/encuestas/ensanut2018/d octos/informes/ensanut_2018_informe_final.pdf

Sharma, B., Cosme, R., y Woo, E. (2018). Prevalence and correlates of insufficient physical activity in school adolescents in Peru. Revista de Saúde Pública, 52(51), 1-13. doi:
https://doi.org/10.11606/S15188787.2018052000202

Slowik, J., Grochowska, E., Maciejewska, I., Kardas, M., Niewiadomska, E., Szostak, M., Irzyniec, T. (2019). Nutritional Status Assessment in Children and Adolescents with Various Levels of Physical Activity in Aspect of Obesity. Obesity Facts, 12(5), 554-563. doi: https://doi.org/10.1159/000502698

Thani, M., Thani, A., Alyafei, S., Kuwari, M., Chetachi, W., Khalifa, S., Akram H. (2018). Prevalence of physical activity and sedentary related behaviors among adolescents: data from the Qatar National School Survey. Public Health, 160 , 150-155.doi: https://doi.org/10.1016/j.puhe.2018.03.019

Whooten, R., Kerem, L.; y Stanley, T. (2019). Physical Activity in Adolescents and Children and Relationship to Metabolic Health. Current Opinion in Endocrinology, Diabetes and Obesity, 26(1), 25-31. doi: https://dx.doi.org/10.1097\%2FMED.000000000 0000455 